\title{
Tolerance and safety of Lactobacillus paracasei ssp. paracasei in combination with Bifidobacterium animalis ssp. lactis in a prebiotic-containing infant formula: a randomised controlled trial
}

\author{
Arine M. Vlieger ${ }^{1}$, Afke Robroch ${ }^{1}$, Stef van Buuren ${ }^{2,3}$, Jeroen Kiers ${ }^{4,5}$, Ger Rijkers ${ }^{6}$, \\ Marc A. Benninga ${ }^{7}$ and Rob te Biesebeke ${ }^{4}$ \\ ${ }^{1}$ Department of Paediatrics, St Antonius Hospital, Nieuwegein, The Netherlands \\ ${ }^{2}$ Department of Statistics, TNO Quality of Life, Leiden, The Netherlands \\ ${ }^{3}$ Department of Methodology and Statistics, FSS, University of Utrecht, The Netherlands \\ ${ }^{4}$ Global Development Centre, Friesland Foods, Leeuwarden, The Netherlands \\ ${ }^{5} \mathrm{NIZO}$, Ede, The Netherlands \\ ${ }^{6}$ Department of Microbiology and Immunology, St Antonius Hospital, Nieuwegein, The Netherlands \\ ${ }^{7}$ Department of Paediatric Gastroenterology, Academic Medical Centre, Amsterdam, The Netherlands \\ (Received 11 September 2008 - Revised 29 January 2009 - Accepted 3 February 2009 - First published online 31 March 2009)
}

The addition of probiotics to infant formula has been shown to be an efficient way to increase the number of beneficial bacteria in the intestine in order to promote a gut flora resembling that of breast-fed infants. The objective of the present study was to evaluate the safety and tolerance of a combination of two probiotic strains in early infancy. A group of 126 newborns were randomised to receive a prebiotic-containing starter formula supplemented with Lactobacillus paracasei ssp. paracasei and Bifidobacterium animalis ssp. lactis or the same formula without probiotics for the first 3 months of life. A total of eighty infants continued the study until they were aged 6 months. Growth measurements were taken monthly at healthy baby clinics. Diaries were used to monitor behaviour, infections, use of antibiotics, as well as stool characteristics. Normal growth occurred in all infants and no statistically significant differences were detected between the probiotics group and the control group for gain in weight, length and head circumference. Infants in the probiotics group produced softer and more frequent stools during the first 3 months of life. No differences were found in crying and sleeping hours, number of parent-diagnosed infections, antibiotic use, visits to the general practitioner and number of adverse events. The use of a prebiotic-containing starter formula supplemented with L. paracase $i$ ssp. paracase $i$ and B. animalis ssp. lactis in early infancy is safe, well tolerated and has no adverse effects on growth and infant behaviour.

Probiotics: Prebiotics: Infant formula: Gut health: Infant health and growth

Breast-feeding is the 'gold standard' in infant nutrition. Therefore, the composition of current infant formulas is largely determined by the composition of human breast milk. Nevertheless, major differences exist between breastfed and formula-fed infants. For example, formula-fed infants have a greater tendency to develop constipation, early wheezing and they also experience more infections in their first year of life ${ }^{(1-3)}$. The composition of the intestinal microbiota differs between formula-fed and breast-fed infants. Breast-feeding stimulates the development of a microbiota dominated by bifidobacteria and lactobacilli, for as much as $90 \%$ of the total gut flora. In contrast, the flora of formula-fed infants is more diverse, containing Bacteroides, bifidobacteria, staphylococci, Escherichia coli and Clostridia ${ }^{(4-6)}$.

The gut flora appears to modulate the health and wellbeing of the host ${ }^{(7)}$ and it has been suggested that the observed differences in microbiota contribute to the lower incidence of infections, allergies and gastrointestinal disturbances in breast-fed infants compared with formula-fed infants ${ }^{(8,9)}$. If so, it seems rational to adapt infant formulas to promote the establishment of an intestinal microbiota resembling that of breast-fed infants. The addition of prebiotics (non-digestible food ingredients) to infant formula has been shown to be an efficient way to increase the number of beneficial bacteria in the intestine ${ }^{(10)}$. Prebiotics are usually well tolerated and regarded as safe. Another approach to improve intestinal microbiota is to add probiotics to infant formula. The addition of $10^{6}-10^{7}$ colony-forming units (CFU) of lactobacilli and/or bifidobacteria per $\mathrm{g}$ of infant milk powder can result in colonisation of the gastrointestinal tract ${ }^{(11,12)}$. Probiotics of bacterial origin are also generally considered safe, but systemic infections with L. rhamnosus and Bacillus have occurred (as summarised by Aggett et al. ${ }^{(13)}$ ). The European Society for Paediatric Gastroenterology, Hepatology and

Abbreviation: CFU, colony-forming units.

* Corresponding author: Dr A. M. Vlieger, fax +31 306094442, email a.vlieger@antonius.net 
Nutrition (ESPGHAN) Committee on Nutrition recommends that infant formulas with added bacteria regarded as probiotics should be marketed only if a full evaluation of benefits and safety has been performed following the general principles as defined by Aggett et al. ${ }^{(13)}$, Koletzko et al. ${ }^{(14)}$ and Agostoni et al. ${ }^{(15)}$.

The purpose of the present study was to assess the safety and tolerance of the addition of Bifidobacterium animalis ssp. lactis (also known as Bifidobacterium Bb-12) as well as Lactobacillus paracasei ssp. paracasei (also known as L. casei CRL-431) to a prebiotic-containing infant formula in a group of healthy, term infants, receiving the formula from birth onwards until the age of 6 months, by identifying any adverse effects, and by examining effects on growth, stooling habits and clinical characteristics such as infections. Bifidobacterium $\mathrm{Bb}-12$ has already been studied extensively in infants. The addition of these species to regular infant feeding has been found to result in normal infant growth with an increase of the number of faecal bifidobacteria ${ }^{(11,16-18)}$. L. casei CRL-431 has shown positive effects in the treatment of diarrhoea in children ${ }^{(19)}$. So far, no safety studies in infants have been performed with $L$. casei CRL-431.

\section{Subjects and methods}

\section{Subjects}

Pregnant mothers, who intended to bottle-feed their infant from birth onwards, were recruited from five antenatal clinics in the central part of the Netherlands. Mothers who stopped breast-feeding within the first week after birth were invited to participate in the study as well. All infants had to be born after $\geq 37$ weeks of gestation and had to be aged $<7 \mathrm{~d}$ at the time of enrolment. Exclusion criteria were the use of antibiotics in the first week, congenital illnesses or malformations that could affect normal growth, and insufficient knowledge of the Dutch language. All parents gave written informed consent. The study protocol was approved by the medical ethics committee of the hospital. This trial is registered as an International Standard Randomized Clinical Trial (no. ISRCTN 78225533).

\section{Trial design}

Infants were randomly allocated using a computerised random-number generator for concealment to either the experimental formula with probiotics or the control formula for the first trimester. Parents of the first eighty infants who completed the first part of the study were asked to continue the use of the study formula for another 3 months. Parents received the assigned infant formula with written instructions for its preparation and were advised to feed infants ad libitum during the study period. Solid foods were introduced at the age of 4 months. Parents were provided with a diary and asked to record crying and sleeping hours, and stool characteristics: frequency of stool passage and consistency (on a four-point scale of $1=$ hard to $4=$ watery and loose) for three consecutive days at the end of each month ${ }^{(20)}$. Parents also recorded the infant's use of antibiotics, visits to their general practitioner, and periods with signs of upper respiratory tract infections and gastrointestinal infections. Furthermore, they were asked to record adverse effects (vomiting, diarrhoea, constipation, colic and rash or eczema). In case of serious adverse events, parents were instructed to contact one of the paediatricians (A. M. V. or A. R.). Anthropometric measurements (length, weight and head circumference) were taken each month at healthy baby clinics. Visits at the hospital took place at 3 months for all infants who completed the first 3 months of the study and at 6 months for those infants who participated also during their second trimester. Furthermore, faecal samples were collected at 1, 2, 3 and 6 months of age for analysis of the faecal flora. The microbiological results will be published separately.

\section{Study formulas}

The experimental and control formulas were standard milkbased powder products (Friso 1; commercially available) that when prepared in accordance with the instructions contained (per $100 \mathrm{ml}$ ): $284 \mathrm{~kJ}, 1.4 \mathrm{~g}$ protein, $1.5 \mathrm{~g}$ fat, $7.3 \mathrm{~g}$ carbohydrates, $0.24 \mathrm{~g}$ prebiotic galacto-oligosaccharides, minerals, vitamins, nucleotides, choline, taurine, L-carnitine and inositol. In addition, the experimental formula contained $1 \times 10^{7}$ CFU B. animalis ssp. lactis/g (also known as Bifidobacterium Bb-12), deposited under American Type Culture Collection (ATCC) number 27536 and $1 \times 10^{7} \mathrm{CFU}$ L. paracasei ssp. paracasei/g (L. casei CRL-431), deposited under ATCC number 55 544. Both formulas had similar taste, smell and colour and were supplied by Friesland Foods, Leeuwarden, the Netherlands. Products were manufactured according to current good manufacturing practices and coded at the manufacturing site. During storage of the product at ambient temperature, the stability of the probiotics in the product was checked monthly by selective plate counting and over a period of 2 years the CFU/g remained stable in the product.

\section{Statistical analysis}

Primary outcomes were differences in growth parameters at 3 months of age. Using data from the Social Medical Survey of Children attending Child Health Clinics (SMOCC) ${ }^{(21)}$, we estimated the weight gain between birth and the age of 3 months equal to 2578 (SD 890) g. We defined the equivalence margin equal to $500 \mathrm{~g}$, and calculated that fifty-five individuals per group were needed for a statistical test using $\alpha=0.05$ and a power $=0.80$. Taking a drop-out rate of $20 \%$ into account, 132 infants had to be enrolled. Secondary outcomes were differences in growth parameters at the age of 6 months, and differences in stool characteristics (consistency and frequency), crying (h/d), the number of upper respiratory tract infections and gastrointestinal tract infections as diagnosed by the parents, the number of antibiotics and visits to the general practitioner.

Diaries and growth charts were analysed by A. M. V., A. R. and S. B. who were all blinded as to the treatment arm. Anthropometric data were expressed as SD scores according to age and sex, with respect to the Dutch references ${ }^{(22)}$. Anthropometric data were checked by plotting the SD score by age of each child. Statistical analyses were performed according to the intention-to-treat principle. Differences between the two therapy groups were analysed by $t$ tests, 
ANOVA, $\chi^{2}$ test and Fisher exact tests where appropriate. SPSS 14.0 (SPSS, Inc., Chicago, IL, USA) and S-PLUS ${ }^{\circledR}$ 8.0 (Insightful Corp., TIBCO Software Inc., Palo Alto, CA, USA) were used for data handling, graphing and statistical analysis.

\section{Results}

Between November 2004 and January 2007, 159 mothers agreed to participate in the study. A flowchart showing the enrolment and status of the infants is presented in Fig. 1. After birth, twenty-six infants were excluded from the study due to practical issues ( $n 5)$, prematurity $(n 4)$, use of antibiotics ( $n$ 3), feeding problems in the first $3 \mathrm{~d}$ ( $n$ 3), refusal of randomisation ( $n$ 2), fear of negative effects $(n$ 2) and unknown reasons $(n 7)$. A total of sixty-nine infants were allocated to the probiotics group and sixty-four to the control group. In the experimental group two infants were lost to follow-up and another fourteen infants dropped out of the study within the first 3 months. In the control group, five infants were lost to follow-up and twelve infants dropped out. Reasons for dropping out were similar in both groups and included, in the experimental and control groups, respectively, colic (6 v. 4), regurgitation ( $1 v .3)$, constipation (3v. 4) and practical issues (4v.1). Thus in total 126 infants (sixty-seven in the probiotics group and fifty-nine in the control group) were considered for the intention-to-treat analysis at 3 months. Since most parents did not fill in the diaries any more after dropping out, only growth parameters could be analysed in the intention-to-treat group. The population for the perprotocol analysis consisted of fifty-three and forty-seven infants at the age of 3 months, and forty-one and thirty-eight infants at the age of 6 months, for the probiotics and control groups, respectively. Upon study entry no differences existed in anthropometric data. However, fewer infants were born by Caesarean section in the probiotics group (Table 1).

\section{Growth}

Table 2 presents the SD scores for weight, length and head circumference at birth and the age of 3 months for the intention-to-treat group, and at the age of 6 months for the perprotocol group. Normal growth occurred in all infants and no statistical differences were detected in SD change scores between the probiotics group and the placebo group for the three growth parameters during the first 3 and 6 months.

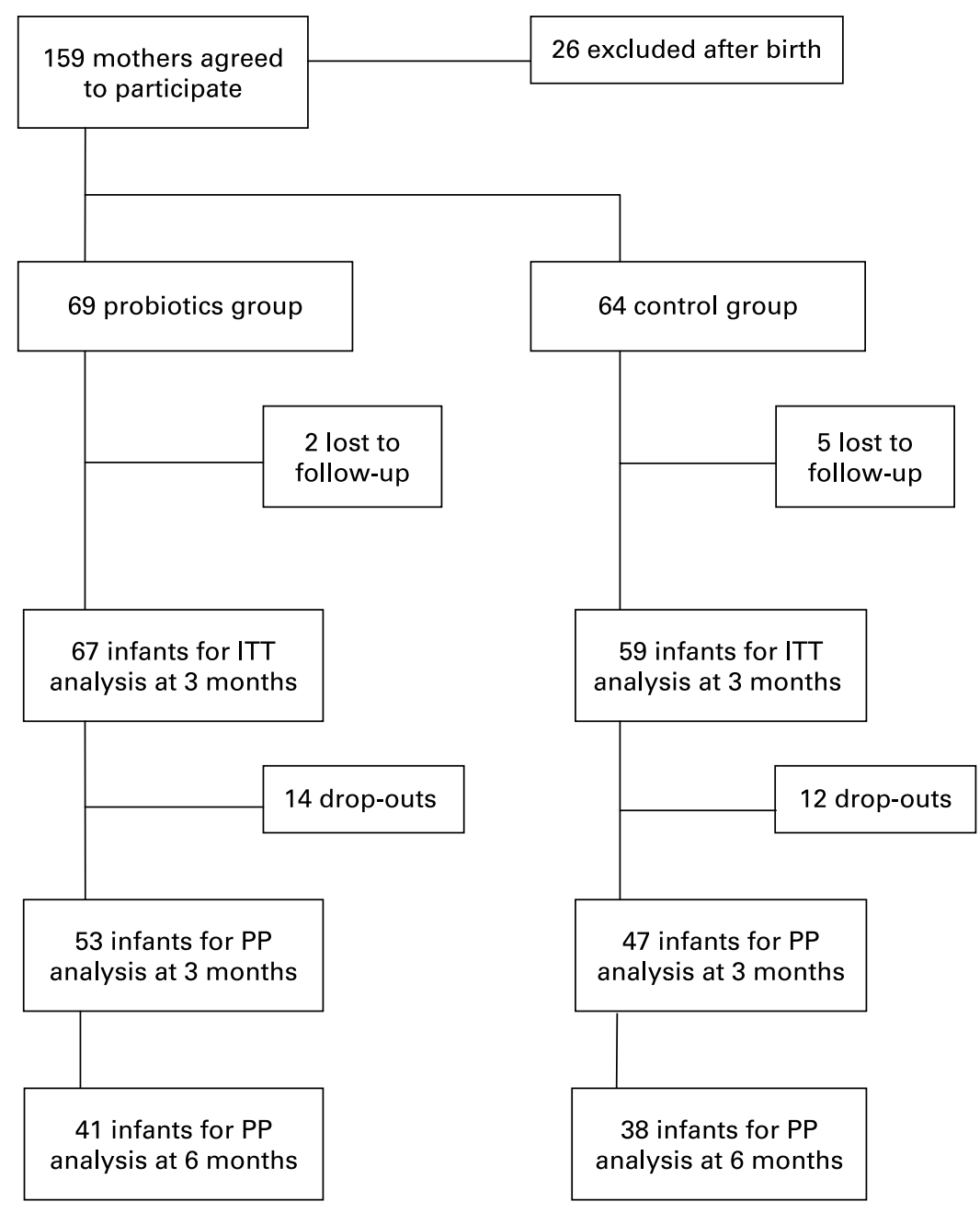

Fig. 1. Trial profile. ITT, intention to treat; PP, per protocol. 
Table 1. Baseline characteristics

(Mean values and standard deviations)

\begin{tabular}{|c|c|c|c|c|}
\hline & \multicolumn{2}{|c|}{ Probiotics ( $n 67)$} & \multicolumn{2}{|c|}{ Control ( $n$ 59) } \\
\hline & Mean & SD & Mean & SD \\
\hline \multicolumn{5}{|l|}{ Infants $(n)$} \\
\hline Males & \multicolumn{2}{|c|}{26} & \multicolumn{2}{|c|}{33} \\
\hline Females & \multicolumn{2}{|c|}{41} & \multicolumn{2}{|c|}{26} \\
\hline Gestational age (weeks) & $40 \cdot 1$ & $1 \cdot 1$ & 39.9 & 1.4 \\
\hline Vaginal delivery $(\%)^{*}$ & \multicolumn{2}{|c|}{97} & \multicolumn{2}{|c|}{88} \\
\hline Birth weight $(\mathrm{g})$ & 3532 & 452 & 3530 & 513 \\
\hline Birth length $(\mathrm{cm})$ & $50 \cdot 6$ & $2 \cdot 2$ & $51 \cdot 1$ & $2 \cdot 5$ \\
\hline Head circumference $(\mathrm{cm})$ & 34.9 & 1.3 & 35.0 & 1.7 \\
\hline \multicolumn{5}{|l|}{ Infants with prior breast-feeding } \\
\hline$n$ & \multicolumn{2}{|c|}{14} & \multicolumn{2}{|c|}{16} \\
\hline Duration of breast-feeding (d) & \multicolumn{2}{|c|}{4.9} & \multicolumn{2}{|c|}{4.6} \\
\hline
\end{tabular}

* Fewer infants were born by Caesarean section in the probiotics group $(P<0.05)$.

The probiotics group gained $2507 \mathrm{~g}$ weight and $10 \cdot 3 \mathrm{~cm}$ length in the first 3 months $v .2661 \mathrm{~g}$ and $10.6 \mathrm{~cm}$ in the placebo group ( $P=0.64$ for weight and $P=0.85$ for length). After 6 months the absolute weight gain was $4152 \mathrm{~g}$ in the probiotics group $v .4282 \mathrm{~g}$ in the placebo group $(P=0.60)$ and gain in length was 17.7 v. $17.3 \mathrm{~cm}(P=0.30)$. Similar results were obtained when growth increments were analysed for each month separately.

\section{Stool characteristics}

Infants in the experimental group had a higher stool frequency during the first 3 months than those in the control group (1.52 v. 1.29 times per $\mathrm{d} ; P=0.04$; Table 3 ). These differences became less pronounced during the second trimester $(1.60 \mathrm{v}$. 1.40 times per $\mathrm{d} ; P=0.13)$. In the first 3 months there was a higher stool consistency-score $(2.57$ v. $2.36 ; P=0.05)$ in the probiotics group, reflecting softer stools. This difference in stool consistency score was not present any more during month 4 to month $6(2.40$ v. $2 \cdot 27 ; P=0.36)$.

\section{Clinical outcomes}

Table 4 shows the variables of general health in the group of infants who completed the study during 6 months. During the intervention no differences were found between the groups regarding crying and sleeping hours, nor in other parameters such as the number of gastrointestinal or upper respiratory tract infections, the amount of antibiotics and visits to the general practitioner. Data analyses on these clinical variables were also carried out for the first 3 months of life and for each month separately; again, results were similar in both groups (data not shown).

\section{Adverse events}

No serious adverse events were reported that could be related to the study formula. Parents were asked if they had noticed any symptoms that could have been caused by the study feed (Table 5). Fewer infants in the probiotics group had developed a rash in the first 3 months (5 v. 12; $P<0.05)$. No differences were seen in other adverse effects between the two groups in both the first and second trimester.

\section{Discussion}

The present study clearly shows that the use of a starter formula containing prebiotics and supplemented with $L$. paracase $i$ ssp. paracasei and $B$. animalis ssp. lactis in early infancy is safe and has no adverse effect on growth and infant behaviour. A higher stool frequency and softer stools were found in those infants receiving the formula containing the probiotic strains compared with infants receiving a standard formula without these probiotics. No significant changes in SD scores for weight, length and head circumference were observed between the study and the control group. All infants had a normal linear and ponderal growth. This is in agreement with similar safety studies with probiotics and synbiotics, in which no negative effect on infant growth was reported ${ }^{(16,23-25)}$. These other safety studies were done with different (combinations of) probiotics. Therefore, the results of these studies cannot simply be extrapolated to other probiotic strains, since different probiotic strains have different metabolic activities.

Drop-out rates, reasons for dropping out and the incidence of adverse events were similar in both the probiotics and the control group, suggesting that the probiotic feeding was well tolerated. We did not find any difference in the incidence of colic or the number of crying hours. This is in contrast to a similar safety study in infants of 3-24 months, using B. animalis ssp. lactis and Streptococcus thermophilus, in which a lower incidence of colic and irritability in the probiotics group was reported ${ }^{(16)}$. The lower age of the infants in the present study and the fact that we used L. paracasei ssp. paracasei instead of $S$. thermophilus may have contributed

Table 2. Growth data expressed as standard deviation score scale*

(Mean values and standard deviations)

\begin{tabular}{|c|c|c|c|c|c|c|c|c|c|c|c|c|}
\hline & \multicolumn{4}{|c|}{ At birth } & \multicolumn{4}{|c|}{ At 3 months } & \multicolumn{4}{|c|}{ At 6 months } \\
\hline & \multicolumn{2}{|c|}{ Probiotics ( $n 67)$} & \multicolumn{2}{|c|}{ Control ( $n 59)$} & \multicolumn{2}{|c|}{ Probiotics ( $n 67)$} & \multicolumn{2}{|c|}{ Control (n 59) } & \multicolumn{2}{|c|}{ Probiotics ( $n 41$ ) } & \multicolumn{2}{|c|}{ Control (n 38) } \\
\hline & Mean & SD & Mean & SD & Mean & SD & Mean & SD & Mean & SD & Mean & SD \\
\hline Weight & 0.22 & $1 \cdot 11$ & 0.14 & 1.23 & 0.34 & 0.75 & 0.39 & 0.99 & 0.10 & 0.72 & 0.17 & 0.89 \\
\hline Length & -0.25 & 1.07 & -0.23 & 1.22 & 0.23 & 0.92 & 0.39 & 1.07 & 0.51 & 0.80 & 0.50 & $1 \cdot 14$ \\
\hline Head circumference & -0.33 & 0.94 & -0.36 & 1.33 & 0.18 & 0.74 & 0.35 & 0.91 & 0.15 & 0.89 & 0.16 & 0.81 \\
\hline
\end{tabular}

${ }^{*}$ There were no statistically significant differences detected between the probiotics and control groups. 
Table 3. Stool characteristics during the first and second trimester

(Mean values and standard deviations)

\begin{tabular}{|c|c|c|c|c|c|c|c|c|}
\hline & \multicolumn{4}{|c|}{ First trimester } & \multicolumn{4}{|c|}{ Second trimester } \\
\hline & \multicolumn{2}{|c|}{ Probiotics ( $n$ 53) } & \multicolumn{2}{|c|}{ Control $(n 47)$} & \multicolumn{2}{|c|}{ Probiotics ( $n$ 41) } & \multicolumn{2}{|c|}{ Control ( $n$ 38) } \\
\hline & Mean & SD & Mean & SD & Mean & SD & Mean & SD \\
\hline Frequency & $1.52^{*}$ & 0.66 & 1.29 & 0.43 & 1.60 & 0.65 & 1.40 & 0.49 \\
\hline Consistency & $2.57^{\star}$ & 0.55 & $2 \cdot 36$ & 0.50 & 2.40 & 0.59 & $2 \cdot 27$ & 0.66 \\
\hline
\end{tabular}

${ }^{*}$ Mean value was significantly different from that of the control group in the first trimester $(P<0.05)$.

to this discrepancy. We did find a lower incidence of rash as reported by the parents in the probiotics group during the first trimester. Since the first medical examination during the study was performed at the age of 3 months, when most of the rashes had disappeared already and were thus self-limiting, we cannot discriminate between the different causes of a rash (viral, allergic, or constitutional) and can only speculate on the reason for this difference. For example, the lower incidence of a rash could reflect an influence of the probiotic strains on the development of atopic eczema, since several studies have reported a reducing effect of probiotic supplementation on the incidence of eczema in atopic infants ${ }^{(26)}$. On the other hand, recently Hol et al. have showed that supplementation of infant formula with similar probiotics did not result in a lower incidence of atopic eczema in a group of infants with cows' milks allergy ${ }^{(27)}$. For these reasons, and because there was no reduction of rash at 6 months, it seems unlikely that the lower incidence of rash at 3 months in the probiotics group in the present study reflects a modulation of the immune system.

Supplementation with $L$. paracasei ssp. paracasei and B. animalis ssp. lactis was associated with an increase in defecation frequency and softer stools during the first trimester. Several other studies in infants have documented similar effects of probiotics or synbiotics on stool frequency and/or consistency $^{(12,24,25)}$. Furthermore, advantageous effects of probiotics on stool consistency and frequency have been reported in constipated children ${ }^{(28,29)}$. Constipation is a frequent symptom in bottle-fed infants with prevalences of up to $17 \%^{(1,30)}$. It would therefore be interesting to explore the significance of our findings in a larger study in infants, adequately powered to examine possible differences in incidence of constipation. Such a study may also help to answer the question whether the presence of prebiotics such as galacto-oligosaccharides are a prerequisite to induce the effect of probiotics on stool consistency and frequency.

Another possibility is to address the effects of the combination of these two probiotic strains on infants who already have developed constipation. The difference in stool characteristics between the two study groups disappeared in the second trimester. We hypothesise that this is due to the fact that the infants started with solid foods in the 4th month, thereby influencing the gut flora significantly. Additional studies, examining the flora of probiotic-fed infants, may help to shed light on this question and also help to unravel the still unknown mechanisms by which probiotics can increase faecal moisture and enhance gastrointestinal motility.

It has been reported that $B$. animalis ssp. lactis reduces the incidence of infectious diarrhoea and the use of antibiotics in infants $^{(16,31,32)}$. Furthermore, L. casei CRL-431 has shown positive effects in the treatment of diarrhoea in children ${ }^{(19)}$. We therefore asked parents to report periods with signs of a gastrointestinal or respiratory infection, use of antibiotics and visits to the general practitioner. In contrast to the above-mentioned studies, we did not find any difference in these parameters between infants with and without probiotics in their formula feeding. The apparent lack of preventive effect by the combination of these two probiotics could be related to the fact that infants in the present trial were much younger than those in the other studies and visited a daycare centre less often, probably resulting in lower risks of developing infections. A longer follow-up study will be necessary to

Table 4. Parameters of general health during the first and second trimester ${ }^{*}$ (Mean values and standard deviations)

\begin{tabular}{|c|c|c|c|c|c|c|c|c|}
\hline & \multicolumn{4}{|c|}{ First trimester } & \multicolumn{4}{|c|}{ Second trimester } \\
\hline & \multicolumn{2}{|c|}{ Probiotics ( $n 53)$} & \multicolumn{2}{|c|}{ Control $(n 47)$} & \multicolumn{2}{|c|}{ Probiotics $(n 41)$} & \multicolumn{2}{|c|}{ Control $(n 38)$} \\
\hline & Mean & SD & Mean & SD & Mean & SD & Mean & SD \\
\hline Crying $(\mathrm{h} / \mathrm{d})$ & 1.8 & $1 \cdot 0$ & 1.8 & 0.9 & 0.9 & 0.7 & 1.0 & 0.9 \\
\hline Sleeping $(h / d)$ & $15 \cdot 3$ & 1.5 & $15 \cdot 2$ & 1.1 & $15 \cdot 0$ & $1 \cdot 2$ & $14 \cdot 6$ & 1.3 \\
\hline Upper respiratory tract infection (times/month) & 0.80 & 0.77 & 0.75 & $1 \cdot 0$ & $1 \cdot 1$ & 0.8 & $1 \cdot 0$ & $1 \cdot 1$ \\
\hline Gastrointestinal infection (times/month) & 0.27 & 0.74 & 0.13 & 0.34 & $0 \cdot 18$ & 0.33 & 0.36 & 0.75 \\
\hline Visits to general practitioner (times/month) & $0 \cdot 25$ & 0.42 & $0 \cdot 27$ & 0.33 & $0 \cdot 28$ & 0.50 & 0.29 & 0.55 \\
\hline Use of antibiotics (times/month) & 0.044 & 0.15 & 0.082 & $0 \cdot 19$ & 0.069 & $0 \cdot 18$ & 0.079 & 0.29 \\
\hline
\end{tabular}

* There were no statistically significant differences detected between the probiotics and control groups. 
Table 5. Number of infants with adverse effects as reported by the parents

\begin{tabular}{lccccc}
\hline & \multicolumn{2}{c}{ First trimester } & & \multicolumn{2}{c}{ Second trimester } \\
\cline { 2 - 3 } & $\begin{array}{l}\text { Probiotics } \\
(n \text { 53) }\end{array}$ & $\begin{array}{l}\text { Control } \\
(n \text { 47) }\end{array}$ & & $\begin{array}{l}\text { Probiotics } \\
(n \text { 41) }\end{array}$ & $\begin{array}{l}\text { Control } \\
(n \text { 38) }\end{array}$ \\
\hline Vomiting & 7 & 9 & & 3 & 6 \\
Diarrhoea & 0 & 1 & & 2 & 0 \\
Constipation & 1 & 3 & & 3 & 4 \\
Colic & 12 & 11 & & 5 & 2 \\
Rash $^{*}$ & 5 & 12 & & 10 & 7 \\
\hline
\end{tabular}

* Fewer infants experienced a rash in the probiotics group compared with in the control group in the first trimester $(P<0.05)$

reveal any protective effects of this combination of probiotics against infectious diseases.

The present study is not without limitations. First, our dropout rate was higher than expected (thirty-three out of 133), resulting in a slightly lower number of children that completed the first treatment period of 3 months than had been calculated in our power analysis. A drop-out rate of $25 \%$ is not uncommon in these kind of studies ${ }^{(16,24,25)}$. It is known that parents often switch formulas in the first 6 months of life (up to $47 \%$ ) because of concerns regarding common infantile symptoms perceived by parents to be related to formula intolerance ${ }^{(33)}$. The decision to switch formula is often made without consulting a health professional. This also occurred in the present study: most of the parents who decided to switch formula did so without notifying the investigators. Since the drop-out rates and the reasons for dropping out in the present study were similar in both treatment groups and because almost all drop-outs occurred in the first 6 weeks, we assume that the higher than expected number of drop-outs was unrelated to the probiotics. Although the study formula was delivered at home at parents' request and parents were repeatedly instructed to use only the study formula, we cannot rule out the possibility that compliance with the protocol was suboptimal, since we did not monitor the intake of the study formula. However, we could confirm the intake of probiotics by faecal analysis, which identified the specific bacterial strains mainly in the probiotics group (F Schuren, AM Vlieger, A Nauta and $\mathrm{R}$ te Biesebeke, unpublished results). Third, we studied the number of infections only by parental observation instead of confirming these infections by examining infants and taking cultures of stools and nose swabs. This could potentially affect the conclusion that the number of infectious periods was similar in both groups. Finally, there were more vaginal deliveries in the probiotics group. It is known that the number of bifidobacteria in the faecal flora is significantly higher after vaginal deliveries in comparison with Caesarean sections ${ }^{(34)}$ and this may have been of influence on the difference in stool consistency and frequency, as found in the present study. Faecal analysis of both infant groups, focusing on these potential differences in bifidobacteria, may answer the questions if this difference in mode of delivery has indeed resulted in a difference in the composition of the flora of both groups and if it was related to changes in stool pattern.

In conclusion, this safety study demonstrates that the administration of a prebiotic-containing starter formula supplemented with $L$. paracasei ssp. paracasei and B. animalis ssp. lactis to infants is safe, well tolerated, does not adversely affect growth and infant behaviour in the short term and can result in softer and more frequent stools.

\section{Acknowledgements}

We thank Marielle Bink and Ralph Witteveen for assistance with data collection.

The study was financed by Friesland Foods, Leeuwarden, The Netherlands.

A. M. V. participated in study design and examination of the infants, coordinated data analysis and interpretation, and was responsible for writing the report. A. R. participated in examination of the infants and data collection. S. v. B. was responsible for data analysis and interpretation. J. K. was responsible for the study design. G. R. participated in data interpretation and writing of the report. M. A. B. contributed to the study design, data interpretation and writing of the report. R. t. B. coordinated the project. All authors have seen and approved the final version.

J. K. and R. t. B. were employed by the sponsor (Friesland Foods, Leeuwarden, the Netherlands) at the time of the study. All other authors declare that they have no conflict of interest.

\section{References}

1. Quinlan PT, Lockton S, Irwin J, et al. (1995) The relationship between stool hardness and stool composition in breast- and formula-fed infants. J Pediatr Gastroenterol Nutr 20, 81-90.

2. Elliott L, Henderson J, Northstone K, et al. (2008) Prospective study of breast-feeding in relation to wheeze, atopy, and bronchial hyperresponsiveness in the Avon Longitudinal Study of Parents and Children (ALSPAC). J Allergy Clin Immunol 122, $49-54$.

3. Wright AL, Bauer M, Naylor A, et al. (1998) Increasing breastfeeding rates to reduce infant illness at the community level. Pediatrics 101, 837-844.

4. Yoshioka H, Iseki K \& Fujita K (1983) Development and differences of intestinal flora in the neonatal period in breast-fed and bottle-fed infants. Pediatrics 72, 317-321.

5. Balmer SE \& Wharton BA (1989) Diet and faecal flora in the newborn: breast milk and infant formula. Arch Dis Child 64, $1672-1677$.

6. Harmsen HJ, Wildeboer-Veloo AC, Raangs GC, et al. (2000) Analysis of intestinal flora development in breast-fed and formula-fed infants by using molecular identification and detection methods. J Pediatr Gastroenterol Nutr 30, 61-67.

7. Guarner F \& Malagelada JR (2003) Gut flora in health and disease. Lancet 361, 512-519.

8. Koletzko B, Aggett PJ, Bindels JG, et al. (1998) Growth, development and differentiation: a functional food science approach. Br J Nutr 80, Suppl. 1, S5-S45.

9. Hanson L \& Telemo E (1997) The growing allergy problem. Acta Paediatr 86, 916-918.

10. Macfarlane S, Macfarlane GT \& Cummings JH (2006) Review Article: prebiotics in the gastrointestinal tract. Aliment Pharmacol Ther 24, 701-714.

11. Langhendries JP, Detry J, Van Hees J, et al. (1995) Effect of a fermented infant formula containing viable bifidobacteria on the fecal flora composition and $\mathrm{pH}$ of healthy full-term infants. $J$ Pediatr Gastroenterol Nutr 21, 177-181.

12. Vendt N, Grunberg H, Tuure T, et al. (2006) Growth during the first 6 months of life in infants using formula enriched with 
Lactobacillus rhamnosus GG: double-blind, randomized trial. J Hum Nutr Diet 19, 51-58.

13. Aggett PJ, Agostini C, Goulet O, et al. (2001) The nutritional and safety assessment of breast milk substitutes and other dietary products for infants: a commentary by the ESPGHAN Committee on Nutrition. J Pediatr Gastroenterol Nutr 32, 256-258.

14. Koletzko B, Ashwell M, Beck B, et al. (2002) Characterisation of infant food modifications in the European Union. Ann Nutr Metab 46, 231-242.

15. Agostoni C, Axelsson I, Braegger C, et al. (2004) Probiotic bacteria in dietetic products for infants: a commentary by the ESPGHAN Committee on Nutrition. J Pediatr Gastroenterol Nutr 38, 365-374.

16. Saavedra JM, bi-Hanna A, Moore N, et al. (2004) Long-term consumption of infant formulas containing live probiotic bacteria: tolerance and safety. Am J Clin Nutr 79, 261-267.

17. Bakker-Zierikzee AM, Alles MS, Knol J, et al. (2005) Effects of infant formula containing a mixture of galacto- and fructo-oligosaccharides or viable Bifidobacterium animalis on the intestinal microflora during the first 4 months of life. Br J Nutr 94, $783-790$

18. Mohan R, Koebnick C, Schildt J, et al. (2006) Effects of Bifidobacterium lactis $\mathrm{Bb} 12$ supplementation on intestinal microbiota of preterm infants: a double-blind, placebo-controlled, randomized study. J Clin Microbiol 44, 4025-4031.

19. Gaon D, Garcia H, Winter L, et al. (2003) Effect of Lactobacillus strains and Saccharomyces boulardii on persistent diarrhea in children. Medicina (B Aires) 63, 293-298.

20. Tjon WE, Ten A \& Wolters M (2004) Infant crying diary: a useful aid in distinguishing between normal and excessive crying behavior (article in Dutch). Ned Tijdschr Geneeskd 148, 257-260.

21. Herngreen WP, van Buuren S, van Wieringen JC, et al. (1994) Growth in length and weight from birth to 2 years of a representative sample of Netherlands children (born in 1988-89) related to socioeconomic status and other background characteristics. Ann Hum Biol 21, 449-463.

22. Fredriks AM, van Buuren S, Burgmeijer RJ, et al. (2000) Continuing positive secular growth change in The Netherlands 1955-1997. Pediatr Res 47, 316-323.
23. Weizman Z \& Alsheikh A (2006) Safety and tolerance of a probiotic formula in early infancy comparing two probiotic agents: a pilot study. J Am Coll Nutr 25, 415-419.

24. Puccio G, Cajozzo C, Meli F, et al. (2007) Clinical evaluation of a new starter formula for infants containing live Bifidobacterium longum BL999 and prebiotics. Nutrition 23, 1-8.

25. Chouraqui JP, Grathwohl D, Labaune JM, et al. (2008) Assessment of the safety, tolerance, and protective effect against diarrhea of infant formulas containing mixtures of probiotics or probiotics and prebiotics in a randomized controlled trial. Am J Clin Nutr 87, 1365-1373.

26. Prescott SL \& Bjorksten B (2007) Probiotics for the prevention or treatment of allergic diseases. J Allergy Clin Immunol 120, $255-262$.

27. Hol J, van Leer EH, Elink Schuurman BE, et al. (2008) The acquisition of tolerance toward cow's milk through probiotic supplementation: a randomized, controlled trial. J Allergy Clin Immunol 121, 1448-1454.

28. Bekkali NL, Bongers ME, Van den Berg MM, et al. (2007) The role of a probiotics mixture in the treatment of childhood constipation: a pilot study. Nutr $J$ 6, 17.

29. Bu LN, Chang MH, Ni YH, et al. (2007) Lactobacillus casei rhamnosus Lcr35 in children with chronic constipation. Pediatr Int 49, 485-490.

30. Infante Pina D, Badia Llach X, Ariño-Armengol B, et al. (2008) Prevalence and dietetic management of mild gastrointestinal disorders in milk-fed infants. World J Gastroenterol 14, 248-254.

31. Chouraqui JP, Van Egroo LD \& Fichot MC (2004) Acidified milk formula supplemented with Bifidobacterium lactis: impact on infant diarrhea in residential care settings. $J$ Pediatr Gastroenterol Nutr 38, 288-292.

32. Weizman Z, Asli G \& Alsheikh A (2005) Effect of a probiotic infant formula on infections in child care centers: comparison of two probiotic agents. Pediatrics 115, 5-9.

33. Nevo N, Rubin L, Tamir A, et al. (2007) Infant feeding patterns in the first 6 months: an assessment in full-term infants. $J$ Pediatr Gastroenterol Nutr 45, 234-239.

34. Penders J, Thijs C, Vink C, et al. (2006) Factors influencing the composition of the intestinal microbiota in early infancy. Pediatrics 118, 511-521. 